\title{
Blindfolded Robotic Teleoperation using Spatial Force Feedback to the Toe
}

\author{
Annette Hagengruber \& Hannes Höppner \& Jörn Vogel
}

\begin{abstract}
This paper examines the capability to incorporate spatial force feedback to the human toe when teleoperating a robotic arm in a force task. Due to the growing complexity of teleoperated systems new means of feedback get increasingly important. To investigate the viability of spatial toe-feedback, experiments with 12 subjects were conducted. The participants had to teleoperate a DLR Light-Weight Robot (LWR) via optical tracking of one finger in order to push a toy train. The orientation of the rail was unknown to the subject and had to be explored using the haptic feedback-a three-dimensional spatial force to the toe, reflecting the contact forces at the robotic endeffector-in absence of visual feedback. The rail was mounted in one of four possible orientations (differences of $45^{\circ}$ ). The main task of the experiment was to identify the present orientation. In our study subjects could successfully identify the orientation of the rail in more than two thirds of all trials $(68 \%)$. In almost half of the trials $(44 \%)$ the subjects were able to move the train along the rails long enough to reach the bumpers at the end and identify them as such. Assuming no feedback would be provided at all, the first metric has a chance level of $25 \%$, and reaching the bumper can be considered impossible. Thus, we can conclude that humans can incorporate spatial force feedback to the toe into their sensorimotor loop.
\end{abstract}

\section{INTRODUCTION}

Modern teleoperated robotic systems allow the user to feel the forces generated when interacting with the environment. For instance the DLR HUG [1], [2], [3]—a multimodal and bimanual telepresence system-allows for intuitive control of a humanoid robot with a head-mounted display and haptic feedback at the user interfaces. However, the number of available force and torque signals (e.g. originating from the different joints of the robotic end-effectors) often is larger than the number of available feedback channels to the user. Take the operation of supernumerary robotic limbs as presented by Llorens-Bonilla et al. [4] as an example: the user manipulates the environment with his own arms and hands while two additional robotic limbs perform supportive tasks. It is not helpful to provide further haptic feedback of the two robotic limbs to the user's arms and hands. Thus, rendering direct haptic feedback is impossible. In other scenarios, like minimally invasive robotic surgery, e.g., with the DLR MIRO system [5], force information from multiple robotic devices may be available. Nevertheless, feedback is only provided to the hands of the surgeon. Thus, to improve controllability of such complex systems, it is necessary to identify new channels to provide the user with intuitive force feedback. It is essential to note, that additional visual input is not applicable as vision is

All authors are with the Institute of Robotics and Mechatronics, German Aerospace Center (DLR), Wessling, Germany often overloaded. It is known, that humans can learn to incorporate feedback from channels which have been lost due to accidents or illness, or even novel kinds of feedback. For example, retinal prosthesis or cochlear implants [6] can successfully restore natural sensory feedback, partly even in spite of never using this feedback channel before. These technologies use existing nerve stimulation in order to restore lost or missing capabilities. Furthermore, new technologies allow to incorporate novel types of feedback. The tongue display unit [7], [8] is a device put on the tongue to provide electrocutaneous stimulation of the anterior-dorsal part of the tongue to feed back sensory information. The display can represent visual information of, e.g., a camera attached to glasses enabling blind people to receive relevant information about their environment. Another technology is the BuzzClip from iMercive Inc., a device for blind or partially sighted people that can be clipped around the upper chest area. It uses ultrasound to detect obstacles and induces vibrations to feed back the occurrence thereof to its wearer. Sparks et al. [9] as well as Eagleman [10] also use vibrotactile feedback, to allow deaf people to perceive audio signals. All these studies and partly commercial available technologies show, that humans can learn to incorporate various kinds of feedback and extract information from it.

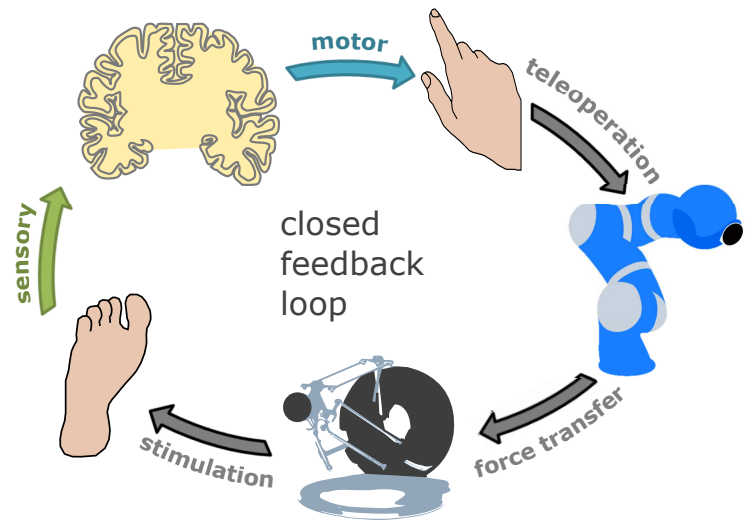

Fig. 1: Schematic illustration of the closed feedback-loop - The human teleoperates the robotic arm in a force task. The force measured at the endeffector is fed back to the user's toe. The perceived sensory information helps to adapt the motor commands in order to fulfill the task.

In this paper, we investigate human capabilities to incorporate force feedback about robotic end-effector forces to the big toe. This sensory loop is particularly relevant since it allows to feed back sensory information from a task scene based on its natural signal modality (force is fed back as force). The idea of feeding back force to the toe is based on 
work by Panarese et al. [11] proposing to use the bare front side of the toe as the feedback receptor. They showed that the mechanoreceptors in the skin of the big toe facilitate the integration of a one-dimensional force-feedback to the sensorimotor system of humans to improve the control over a robotic hand. Their research proves the basic concept, i.e. humans are able to close the loop between motor functionality provided by the hand and sensory information given to the toe (see Fig. 1). We hypothesize that this type of feedback also allows for a fast and reliable incorporation of spatial three-dimensional force feedback, consisting of normal and tangential force components. Panarese and Edin [12] showed that the mechanoreceptors of the glabrous skin of the fingertips enable the discrimination of spatial forces. However, the incorporation of spatial, i.e. three-dimensional, feedback at the toe has not yet been investigated.

The paper is structured as follows: In Section II we will explain the the experimental setup and design, as well as the data acquisition and analysis. Section III presents the results of 12 subjects performing the experimental protocol. The paper is closed with Section IV discussing and concluding the obtained results.

\section{METHODS}

To validate that the human is able to incorporate spatial force feedback to the toe into the sensorimotor loop during tasks requiring hand and arm motion, we choose a complex manipulation task. The experiment is designed such, that a pure feed-forward approach without visual or force feedback will lead to no or very little success.

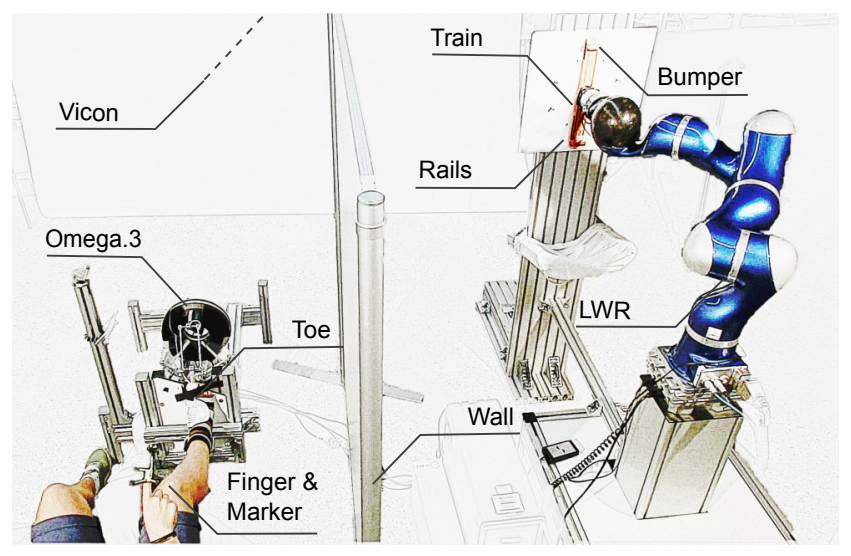

Fig. 2: Experimental Setup - The subject on the left can control the position of the robot end-effector through the optical tracking marker. The right big toe of the subject is in contact with the tip of the Omega. 3 force feedback device. The robot is located out of view for the subject (right hand side) and pushes a wooden train on a rail.

In this task, subjects have to teleoperate the LWR in order to push a wooden toy train $(\mathrm{BRIO} \cap \mathrm{GmbH})$ against a straight rail. The rail is mounted upright on a wall and can be reoriented in four different orientations, but the very orientation is unknown to the subject. The endpoints of the rail are equipped with bumpers, which the train can't pass. The goal of the task is A) to determine the orientation of the rail and B) to identify one of the two bumpers of the rail, when reaching one with the train. The expectation of correct orientation classification (task A) by guessing at chance is at $25 \%$. Task B, however, requires precise control of the robot's force and position and is almost impossible to solve without feedback, even if the orientation was known.

\section{A. Experimental Setup and Feedback Description}

The experimental setup is depicted in Fig. 2. The BRIO rail is mounted on a vertical wall, such that the train immediately falls down when not pushed against the rail. Force is applied to the train using an LWR operated in Cartesian impedance control [13]. On the one hand, this type of controller provides stability when operating in contact with rigid objects. On the other hand, it allows to apply defined forces to the robotic end-effector. The translational position of the robotic end-effector is directly controlled by the subject via optical tracking of a passive marker attached to the index finger. The optical tracking signal is captured using a windows machine and sampled at $100 \mathrm{~Hz}$. The software for controlling the setup is implemented in

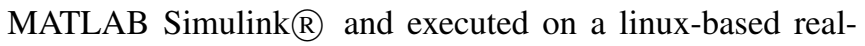
time computer running at $1 \mathrm{kHz}$ sample frequency. In order to generate a smooth motion of the robot, we use the interpolation algorithm introduced in [14]. The end-effector orientation is constant. During the experiment subjects have no visual feedback of the task scene as it is occluded by a partition wall. Thus, except for the toe-feedback, this set-up allows teleoperation with neither tactile nor visual information provided to the subject.

As the robot is occluded during the experiments, it automatically holds the train against the rail before the subject gets control over its position $\mathbf{x}_{\mathbf{d}}$. External forces that act on the LWR and the feedback device are eliminated before the task. The weight of the train of $47 \mathrm{~g}$ deliberately has been kept low. We assume that the tangential force resulting by the weight of the train is negligible compared to the force needed to overcome the friction. While pushing the train against the rail, the direction thereof constrains the train's actual motion $\mathbf{x}_{\mathbf{m s r}}$. The applied force is linearly coupled to the position of the robot via the stiffness $\mathbf{k}$. Only taking into account the translational component of the impedance control law, the force $\mathbf{F}$ resulting at the end-effector of the robot is:

$$
\mathbf{F}=\mathbf{k}\left(\mathbf{x}_{\mathbf{d}}-\mathbf{x}_{\mathbf{m s r}}\right) \text {. }
$$

Moving the robot closer to the rail, the force pushing the train on the rail increases. Motion sideways to the rail direction leads to an increase in shear-force, which ultimately will push or tilt the train off the rail. Motion in the longitudinal direction of the rail will move the train along the rail. In order to precisely acquire the forces exerted on the train, a force-torque sensor is mounted at the robotic end-effector.

These measured forces are applied to the subjects right big toe using an Omega.3 device by Force Dimension as depicted in Fig. 3. This device is a delta-based parallel kinematic with active gravity compensation. It provides three translational 


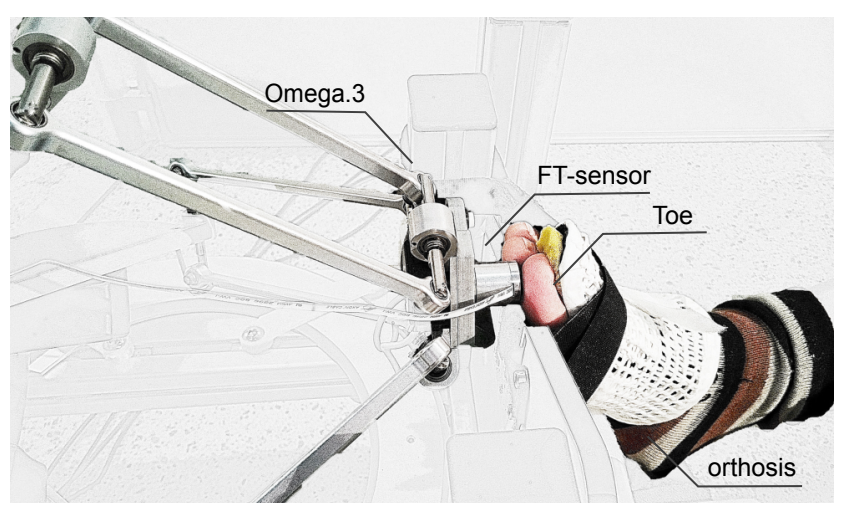

Fig. 3: Experimental Setup, a closeup view of the toe - The right toe is fixed in front of the haptic feedback device. The device stimulates the bare front side of the distal phalanx of the toe.

degrees of freedom and reaches a cylindrical work space with a diameter of about $\varnothing 160 \mathrm{~mm}$ and length of $110 \mathrm{~mm}$. It provides a maximum force of $20 \mathrm{~N}$ and a stiffness of $14.5 \mathrm{~N} / \mathrm{mm}$ [15]. For realizing precise force feedback to the toe, we use the DLR Fingertip sensor mounted on the tip of the Omega.3. The additional sensor in combination with a PID controller facilitates precise control of the forces applied to the toe. For optimal skin connection a hemispheric plastic tip with comparably high stiffness (in relation to the stiffness of human skin) and a diameter of $10 \mathrm{~mm}$ is mounted on the force sensor. The force controlled Omega. 3 device allows to stimulate spatial forces of up to $12 \mathrm{~N}$ at a maximum frequency of $5 \mathrm{~Hz}$.

The contact between toe and tip of the feedback device depends on friction. To prevent slip of the feedback device, we restrained motion of the toe using a orthosis which is fixed to the base of the Omega.3. Furthermore, we limit the tangential components $F_{T}$ of the feedback force not to exceed 0.3 times the normal force $F_{N}$. This corresponds to a friction angle of $\sim 17^{\circ}$. Given the limitation of the normal force of $12 \mathrm{~N}$, we set the robotic stiffness $\mathrm{k}$ in Eq. (1) to $600 \mathrm{~N} / \mathrm{m}$ in the normal direction. This results in a range of motion of $2 \mathrm{~cm}$ normal to the rail, i.e. between full force and dropping the train. For the tangential components, we choose a stiffness factor of $800 \mathrm{~N} / \mathrm{m}$ to increase responsiveness in the tangential directions. An auditive cue informs the subject whenever the normal force limit of $12 \mathrm{~N}$ is reached. While the robot could be commanded to push stronger, no increase in the toe feedback is possible beyond this limit.

Besides this audio cue, the force feedback to the toe is the only feedback provided to the subjects. Thus, the subject has to extract information about the current state of the train on the rail from the toe feedback. Further, it has to identify the orientation of the rail by minimizing tangential forces perceived at the toe. During the experimental procedure no feedback about success is given to the subjects even after accomplishing a trial.

\section{B. Experimental Design}

During the experiment, the orientation of the BRIO rails is randomly selected from a set of four different directions. Available orientations (see Fig. 4) are:

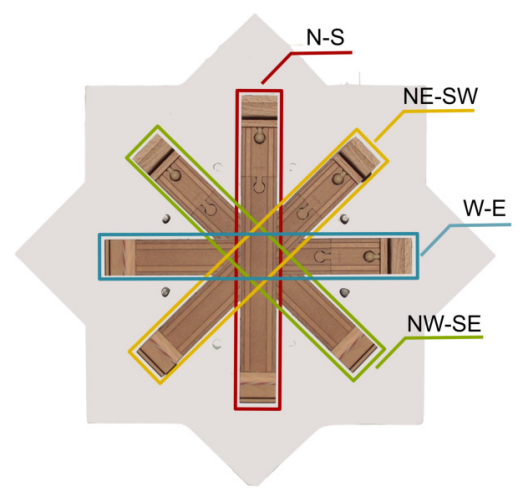

Fig. 4: Rail-orientations - The four available orientations that the rails could have during the experiments: North-South (N-S), Northeast-Southwest (NE-SW), West-East (W-E), item Northwest-Southeast (NW-SE).

- North-South (N-S)

- Northeast-Southwest (NE-SW)

- West-East (W-E)

- Northwest-Southeast (NW-SE)

The goal of the task is to identify how the rails are oriented. Subjects should use the occurring normal (pushing force) and tangential forces to successfully move the train along the rail and thereby identify the rail-orientation. Moving the train contrary to the given rail-orientation leads to higher tangential forces at the robot end-effector and consequently at the toe.

Additionally to identify the orientation of the rail, the subjects are asked to drive the train along the rails until one, or if possible, both bumpers are hit. The subjects disclose their perception about the rail-orientation (measure $C-$ correctly identified rail orientation or not) to the experimenter recording the results. Additionally, the experimenter records whether none, one, or both bumpers have been reach in a trial.

The main experiment consists of 20 trials; each railorientation is repeated five times in randomized order. The procedure for a single trial is as follows: The subject chooses a convenient arm/finger position to start teleoperation. The train is placed in the center of the rails and the robot automatically moves forward to apply an initial normal force and pushes the train on the rail. The subject perceives this initial force as feedback to the toe. An audio cue signal indicates the subject the start of the teleoperation phase. Afterwards, the robot follows the motion of the subject's index finger. Given that the train starts in the middle of the rail, subjects can potentially move in two directions (e.g., up or down on the N-S rail).

The trial stops either when the subjects report to have reached both bumpers of the rail once (even when this is not the case) or if the train is dropped. The train drops, when the robot is moved in a wrong direction and consequently leaves the rails, or beyond the end of the rail. Dropping of the train will also happen when the normal force is too low. As soon as the trial ends, subjects have to report the direction of the rail. Intentionally, we do not put any time pressure on the subjects allowing them to take as much time as they need.

As force feedback to the toe is a new tactile perception 
for subjects, a training phase is inevitable. The training procedure is conducted prior to the 20 trials and includes three phases. In the first phase subjects are allowed to watch the robot while they are teleoperating it. Only during this phase subjects have both, visual and force feedback of the task scene. Within this phase, each of the four different orientations of the rail is trained for one minute. In case the train is dropped during training, the experimenter resets the task, and the training can be continued.

In the second training phase, visual feedback is removed by setting up a partition wall between subject and robot. Again, all four directions are presented to the subject for about one minute, while the subject is aware of the actual orientation. Also in this phase, training may be continued after dropping the train.

The third phase is equal to the main experiment, except that feedback about success is given by the experimenter. However, this phase only consists of four trials, one for each possible rail orientation. While the stopping conditions are the same as in the experimental part, at the end of each training trial the experimenter informs the subject whether the bumpers have been successfully reached and whether the perceived direction corresponds to the actual direction of the rail.

After training, the subjects receive no more feedback from the experimenter. Subsequent to each training phase, and after trial 7 and 14, subjects are given a two minute break. At the end of the main experiment, the subjects have to fill in a questionnaire about their mental demand, their perceived performance, frustration, and comfort they have felt during the experiment. This rating is a scale between 0 and 20, with 0 being worst and 20 best.

\section{Participants}

A total of 12 healthy subjects including 11 men and 1 woman, aged 23-35 years, performed the experimental protocol as described above. No subject reported a history of neurological disorder or neuromuscular injury. All subjects gave written consent to the procedures which were conducted mostly in accordance with the principles of the Helsinki agreement ${ }^{1}$. Before starting the experiments, subjects were briefed by describing the experimental procedure and the goal of the experiment. During the tests the subjects were sitting in front of the feedback device so that their rightbig toe could be stimulated and their right hand and arm was free to move. All subjects were novices and have never performed similar experiments before.

\section{Data Acquisition and Analyses}

The experiment is designed so as to present each railorientation to each subject five times. The experimenter records the subjects responce about rail-orientation (measure $C$ ) and the acquired rail-endings (measure $E$ ). Furthermore, data from the robot including $\mathbf{x}_{\mathbf{d}}, \mathbf{x}_{\mathbf{m s r}}$, and the time is

\footnotetext{
${ }^{1}$ Non-conformity concerns point B-16 of the 59th World Medical Association Declaration of Helsinki, Seoul, October 2008: no physician supervised the experiment.
}

digitally recorded. This data is used to calculate the mean time, that a subject needs to reach the first bumper $\left(\bar{T}_{A}\right)$.

From the data of the force-torque sensor at the end-effector of the robot and of the Omega. 3 we calculate $\bar{T}_{T}$. This is the mean time the subjects exceeded the normal force limit of $12 \mathrm{~N}$ within a trial until the first bumper is reached. The later condition is set to make the times comparable. Further, we calculate the mean normal and tangential forces $\left(\bar{F}_{N}, \bar{F}_{T}\right)$ of the force-torque senor at the end-effector. Unfortunately, two data sets were lost during the experiments: the forcetorque sensor data of subject 5 and subject 9 . Hence, it is not possible to calculate $\bar{F}_{N}$ and $\bar{F}_{T}$ for these two subjects.

The correct answers of $C$ are sorted by direction to perform a one-way repeated measures ANOVA on the data. This statistical test is executed with the statistics and machine learning toolbox in MATLAB.

\section{RESULTS}

The main task for the subjects was to interpret the haptic toe-feedback in order to recognize the given orientation of the rails. On average, $68 \%$ of the orientations were successfully identified by the participants. The probability to choose the right rail-orientation by chance is $25 \%$. Thus, identification rate over all subjects exceeds this chance-level by more than double. In $44 \%$ of all trials the subjects were able to identify at least one of the two bumpers and in $15 \%$ of the cases even both. In these 38 trials (15\%) the subjects were able to move the train along the rails without dropping it. Arguably, it is almost impossible to move the train along the rail without any feedback.

There are small differences between the four different railorientations. While on average $3.4( \pm 1.37)$ correct answers are given in the orientations of N-S, $3.9( \pm 0.99)$ in NE$\mathrm{SW}$, and $3.8( \pm 0.88)$ in NW-SE, the W-E orientation is only correctly identified $2.66( \pm 1.15)$ times by the subjects on average. A one-way repeated measures ANOVA for the factor rail-orientation showed a significant difference $(\mathrm{p}=0.039, \mathrm{~F}=$ 3.12 , d.f. $=3$ ) with respect to the measure $C$. Fig. 5 visualizes the amount of correct answers per rail-orientation.

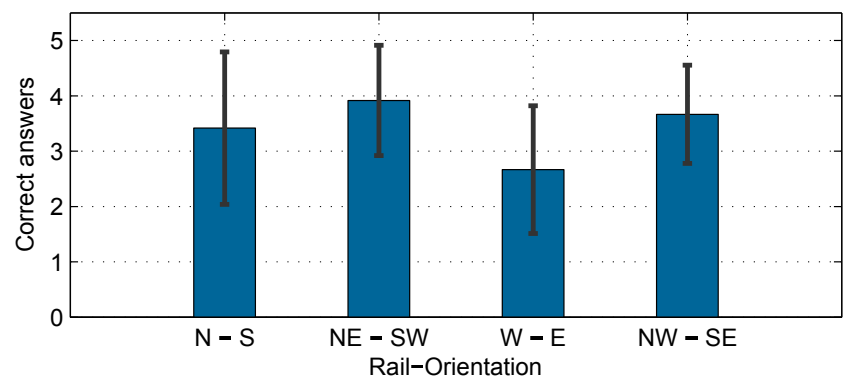

Fig. 5: Correct answers on average - The bars show the mean and the standard deviation of the correct answers per rail-orientation. The orientation $\mathrm{W}-\mathrm{E}$ was identified slightly worse in comparison to each of the other.

We further analyzed the time needed to reach a bumper. The duration averages between 36 and $38 \mathrm{sec}$. for the orientations NE-SW, W-E and NW-SE. Contrary, in the N-S 


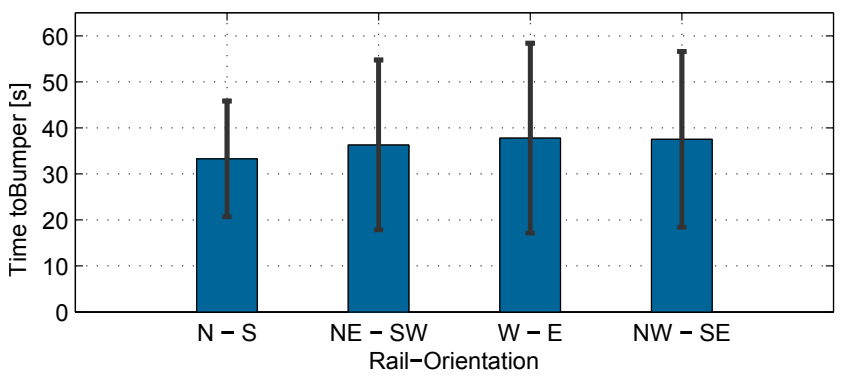

Fig. 6: Needed time to rail-ending - The bars show the mean time plus standard deviation that the subjects needed to reach the first ending. The $\mathrm{N}-\mathrm{S}$ orientation showed lower times with an average of 33.2 seconds.

\begin{tabular}{|c||c|c|c|c|c|c|}
\hline$\#$ & $\mathbf{C}$ & $\mathbf{E}$ & $\bar{F}_{N}[\mathbf{N}]$ & $\bar{F}_{T}[\mathbf{N}]$ & $\bar{T}_{A}[\mathbf{s e c}]$ & $\bar{T}_{T}[\mathbf{s e c}]$ \\
\hline \hline $\mathbf{1}$ & 11 & 13 & 7.3 & 1.6 & 16.8 & 2.7 \\
\hline $\mathbf{2}$ & 14 & 9 & 5.3 & 1.0 & 33.5 & 0.7 \\
\hline $\mathbf{3}$ & 11 & 5 & 5.3 & 1.4 & 52.3 & 0.9 \\
\hline $\mathbf{4}$ & 12 & 7 & 7.0 & 1.5 & 36.4 & 3.2 \\
\hline $\mathbf{5}$ & 18 & 12 & - & - & 43.8 & - \\
\hline $\mathbf{6}$ & 13 & 10 & 6.5 & 1.5 & 42.2 & 4.8 \\
\hline $\mathbf{7}$ & 19 & 13 & 11.3 & 2.1 & 44.4 & 31.3 \\
\hline $\mathbf{8}$ & 12 & 8 & 7.9 & 1.5 & 32.0 & 0.62 \\
\hline $\mathbf{9}$ & 15 & 9 & - & - & 25.1 & - \\
\hline $\mathbf{1 0}$ & 12 & 7 & 7.5 & 1.6 & 28.9 & 0.34 \\
\hline $\mathbf{1 1}$ & 13 & 7 & 8.6 & 1.5 & 56.2 & 7.2 \\
\hline $\mathbf{1 2}$ & 14 & 6 & 7.5 & 2.1 & 30.6 & 1.5 \\
\hline \hline mean & 13.6 & 8.8 & 7.4 & 1.6 & 36.9 & 5.6 \\
\hline \pm std & 2.6 & 2.7 & 1.7 & 0.3 & 11.4 & 9.3 \\
\hline
\end{tabular}

TABLE I: Results of the individual subjects $-\mathrm{C}$ : correct answers out of 20 trials; E: at least one bumper reached; $\bar{F}_{N}$ : mean normal force until reaching the first bumper; $\bar{F}_{T}$ : mean tangential force until reaching the first bumper; $\bar{T}_{A}$ : mean time required to reach the first bumper; $\bar{T}_{T}$ : mean time of force saturation within the time of reaching the first bumper.

orientation it only takes $33.2 \mathrm{sec}$ on average. These results are depicted in Fig. 6.

Table I summarize all measures introduced in Section II for each individual subject. They differ strongly in the amount of correct answers, the time needed, as well as in the force applied during the trials. The listed forces $\left(\bar{F}_{N}\right.$, $\left.\bar{F}_{T}\right)$ and times $\left(\bar{T}_{A}, \bar{T}_{T}\right)$ refer to the time interval from the beginning of a trial until reaching the first bumper. The table describes the mean values per subject over the trials. To ensure better comparability of the values, trials where no bumper has been reached are not considered here.

Correctly identified directions per subjects vary between 11 and 19 out of 20 trials. Moreover, the amount of reached bumpers scatters between 5 and 13 per subject. On average, the normal force is about $7.4 \mathrm{~N}$ but it does not show a significant correlation with the correct answers. Since the tangential forces $F_{T}$ depend on the normal force $F_{N}$ (due to the friction taper), the mean tangential forces $\bar{F}_{T}$ are clearly smaller in comparison to $\bar{F}_{N}$.

It is interesting to note that Subject 7 performed best with 19 correct answers for the 20 trials. For this subject the mean forces $\bar{F}_{N}$ and $\bar{F}_{T}$ show the highest values among the subjects. Despite the audio cue, signaling that the limit of $F_{N}$ has been reached, subject 7 often increased $F_{N}$ up to this limit and exceeded it. Consequently, this lead to higher values for the tangential forces $\bar{F}_{T}$.

The different normal and tangential forces, as well as, the different timings of the participants show that the subjects used different strategies to perform the task. Fig. 7 exemplary depicts two trials from different subjects. Variable $\mathbf{x}_{\mathbf{d}}$ (in grey) shows the desired motion of the robot, i.e. how the robot would move if there was no resistance to the robotic end-effector. However, the pressure against the train on its rails constrains the motion of the robot, resulting in the actual motion $\mathbf{x}_{\mathbf{m s r}}$ depicted in blue. This deviation from the desired motion, in combination with the impedance control generates the forces fed back to the subject's toe (cf. Eq. 1). In the beginning of the trial depicted in subfigure A, the subject tried different directions of motion and started to move in the direction of lowest tangential force. Contrary, the subject shown in subfigure B had a shorter initial testing phase and continuously ensured that the selected direction was correct when tracing the path.

The results show no significant learning effects between the first and second half of the 20 trials for each subject. Neither for the amount of correct answers nor the time needed to reach an end stop. The questionnaire filled in by each subject revealed that they felt an average mental demand for fulfilling the task (11.25 points of 20 over all subjects). Their frustration level during the task and their self-assessment about their performance was also centered with 10.25 and 10.92 out of 20 points. The comfort during the experiments was rated with 14.82 points out of 20 .
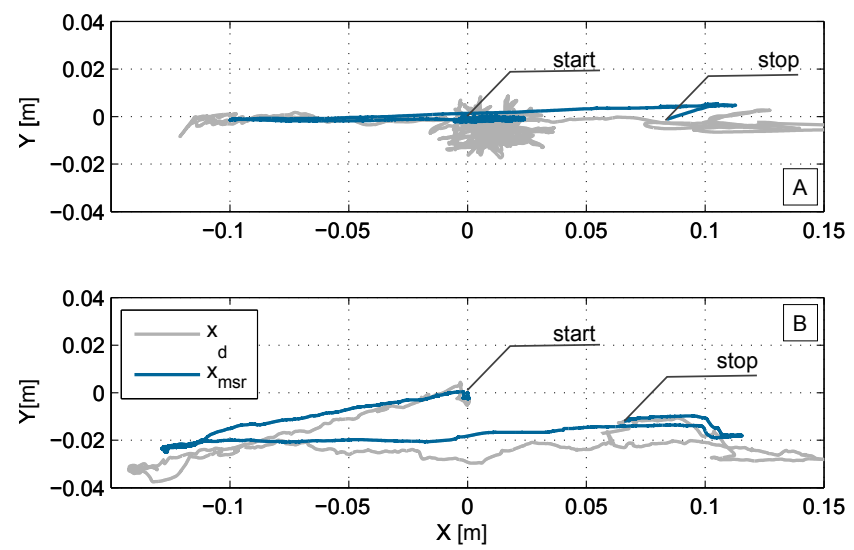

Fig. 7: Examples of the W-E task - Both trials show the W-E orientation which was recognized by both subjects. $\mathbf{x}_{\mathbf{d}}$ shows the path the subjects commanded with their finger. The pressure against the rails generated forces that were fed back to the subjects' toes. $\mathbf{x}_{\mathbf{m s r}}$ show how the end-effector moved along the rails. Upper (A): an example of subject 7; Below (B): an example of subject 3 .

\section{DISCUSSION AND CONCLUSION}

Subjects are able to incorporate spatial force feedback provided to the big toe to successfully teleoperate a robotic arm using optical tracking of their finger tip. This finding is backed by the evidence that on average two thirds of all trials are successfully identified over all subjects. This result is considerably higher than the one fourth of correct answers to be expected by chance. Due to the similar physiology structure of the bare front site of the toe in comparison to the finger tips, this result is not unexpected. The glabrous skin of the hand offers four different types of mechanoreceptors, 
i.e. two fast adapting (FA I, FA II) and two slow adapting receptors (SA I, SA II) [16]. Contrary to the hairy skin, this constellation and distribution of these mechanoreceptors is only available in glabrous skin. Kennedy et al. [17] demonstrate that all four mechanoreceptors, known to exist in the glabrous skin of the hand, are available in the glabrous skin of the foot sole, as well. A particularly sensitive region of the foot sole is the big toe since three of the four mechanoreceptors are available (SA I, SA II, FA I). Therefore, it would be interesting to compare the findings of this paper with spatial force feedback to the fingers in a contrastable task.

While there is potential for improvement $(68 \%$ correct answers) it is essential to note that the subjects had almost no training on the given task. The entire training phase took about ten minutes per subject. During this time, first the subject had to get acquainted with teleoperating the position of the robot via tracking of the finger. Furthermore, the subject had to learn to apply the appropriate amount of normal force to hold the train on the rail. Finally, one had to get a feeling for the differences in orthogonal (increasing $F_{T}$ ) and longitudinal (small $F_{T}$ ) forces and their relation to the state of the train on the rail.

Ideally, the subject would also develop a strategy how to evoke appropriate forces without dropping the train and thereby solve the task. Given the complexity of the task, we hypothesize that the training time was comparably short and further training could improve the performance. In contrast to other types of feedback-e.g., vibrotactile or electrostimulating feedback - the force feedback to the toe provides the sensory information in the same modality (force to force) which might be one reason for the experimental success regarding the short training period. This feedback-modality actually provides an intuitive possibility to incorporate the feedback-control in robotic teleoperation. The subjects in our experiments received no information about success during the task, even after finishing a trial, which sometimes led to frustration. Nevertheless, the toe-feedback helped them to successfully identify at least one of the two bumpers in almost half of the cases, which can be considered impossible without any feedback.

Statistical analysis revealed a significant influence of rail orientation on the result. A post-hoc test proofed that the W-E orientation shows significantly worse results compared to the NE-SW orientation. Further disparities could be noticed in execution time (less in N-S). Due to the complexity of the task, it is not clear whether these findings are caused by the physiology of the toe, the complexity of task itself, or if the weight of the train plays a larger role than expected. Thus, future studies are needed to investigate this effect.

Furthermore, we found that the subjects with the best results often increased $F_{N}$ up to the device saturation of $12 \mathrm{~N}$. As a result $F_{T}$ also reaches higher values. This could be a benefit for interpreting the occurring tangential forces, respectively the events at the end-effector. Future studies have to clarify how strong the influence of the amplitude of tangential force is and how strong they should be to ensure a positive incorporation. Therefore, it is needed to increase the friction angle, in order to test a larger variety of tangential forces. To achieve this, a different kind of linkage between toe and feedback device should be evaluated. This study was a proof of concept of integration and interpretation of spatial force feedback to the big toe. Further studies could validate if also more complex tasks are possible with this approach and the reason for the differences in the rail orientations.

\section{ACKNOWLEDGMENT}

We would like to thank Rachel Hornung for her valuable input to this manuscript as well as all our subjects.

\section{REFERENCES}

[1] J. Artigas, P. Kremer, C. Preusche, and G. Hirzinger, "Testbed for telepresent on-orbit satellite servicing," in Proceedings of the HumanCentered Robotic Systems Conference (HCRS), 2006.

[2] P. Kremer, T. Wimböck, J. Artigas, S. Schätzle, K. Johl, F. Schmidt, C. Preusche, and G. Hirzinger, "Multimodal telepresent control of dlr's rollin' justin," in Robotics and Automation, 2009. ICRA '09. IEEE International Conference on, May 2009, pp. 1601-1602.

[3] T. Hulin, K. Hertkorn, P. Kremer, S. Schätzle, J. Artigas, M. Sagardia, F. Zacharias, and C. Preusche, "The DLR bimanual haptic device with optimized workspace," in Robotics and Automation (ICRA), 2011 IEEE International Conference on, May 2011, pp. 3441-3442.

[4] B. Llorens-Bonilla, F. Parietti, and H. H. Asada, "Demonstrationbased control of supernumerary robotic limbs," in 2012 IEEE/RSJ International Conference on Intelligent Robots and Systems. IEEE, 2012, pp. 3936-3942.

[5] U. Hagn, M. Nickl, S. Jörg, G. Passig, T. Bahls, A. Nothhelfer, F. Hacker, L. Le-Tien, A. Albu-Schäffer, R. Konietschke et al., "The DLR MIRO: a versatile lightweight robot for surgical applications," Industrial Robot: An International Journal, vol. 35, no. 4, pp. 324336, 2008.

[6] W. F. House, "Cochlear implants." The Annals of otology, rhinology, and laryngology, vol. 85, no. 3Pt2, p. 1, 1976.

[7] "The tongue display unit (tdu) for electrotactile spatiotemporal pattern presentation," Scientia Iranica, vol. 18, no. 6, pp. 1476 - 1485, 2011.

[8] P. Bach-y Rita, K. A. Kaczmarek, and M. E. Tyler, "A tongue-based tactile display for portrayal of environmental characteristics," Virtual and adaptive environments, pp. 169-186, 2003.

[9] D. W. Sparks, P. K. Kuhl, A. E. Edmonds, and G. P. Gray, "Investigating the mesa (multipoint electrotactile speech aid): The transmission of segmental features of speech," The Journal of the Acoustical Society of America, vol. 63, no. 1, pp. 246-257, 1978.

[10] D. Eagleman, "Plenary talks: A vibrotactile sensory substitution device for the deaf and profoundly hearing impaired," in 2014 IEEE Haptics Symposium (HAPTICS). IEEE, 2014, pp. xvii-xvii.

[11] A. Panarese, B. Edin, F. Vecchi, M. Carrozza, and R. Johansson, "Humans can integrate force feedback to toes in their sensorimotor control of a robotic hand," Neural Systems and Rehabilitation Engineering, IEEE Transactions on, vol. 17, no. 6, pp. 560-567, 2009.

[12] A. Panarese and B. B. Edin, "Human ability to discriminate direction of three-dimensional force stimuli applied to the finger pad," Journal of neurophysiology, vol. 105, no. 2, pp. 541-547, 2011.

[13] A. Albu-Schäffer, C. Ott, and G. Hirzinger, "A unified passivitybased control framework for position, torque and impedance control of flexible joint robots," The International Journal of Robotics Research, vol. 26, no. 1, pp. 23-39, 2007.

[14] R. Weitschat, A. Dietrich, and J. Vogel, "Online motion generation for mirroring human arm motion," in 2016 IEEE International Conference on Robotics and Automation (ICRA). IEEE, 2016, pp. 4245-4250.

[15] force dimension, force dimension user manual omega.x haptic device, version 1.7 ed., 2013.

[16] R. S. Johansson and Å. B. Vallbo, "Tactile sensibility in the human hand: relative and absolute densities of four types of mechanoreceptive units in glabrous skin." The Journal of physiology, vol. 286, no. 1, pp. $283-300,1979$.

[17] P. M. Kennedy and J. T. Inglis, "Distribution and behaviour of glabrous cutaneous receptorsin the human foot sole," The Jornal of Physiology, 2002. 